Check for updates

Cite this: Phys. Chem. Chem. Phys., 2019, 21, 3742

Received 18th October 2018 Accepted 15th January 2019

DOI: $10.1039 / \mathrm{c} 8 \mathrm{cp} 06509 \mathrm{~h}$

rsc.li/pccp

\title{
From nano-emulsions to phase separation: evidence of nano-segregation in (alkane + perfluoroalkane) mixtures using ${ }^{129} \mathrm{Xe}$ NMR Spectroscopy $\dagger$
}

\author{
Pedro Morgado, (D) ${ }^{a}$ Luís F. G. Martins (D) ab and Eduardo J. M. Filipe (D) *a
}

\begin{abstract}
In this work we demonstrate that mixtures of (hexane + perfluorohexane) above the upper critical solution temperature segregate by forming domains at the nanometric scale. ${ }^{129} \mathrm{Xe} N M R$ spectra obtained for solutions of xenon in liquid mixtures of (hexane + perfluorohexane) as a function of temperature suggest the existence of domains richer in the hydrogenated component, in which xenon "prefers" to be solvated. The average local concentration within the xenon coordination sphere is at least 0.05 higher in hexane mole fraction than the nominal concentration of the mixture. Atomistic molecular dynamics simulations support this analysis in excellent agreement with the experimental data. Additionally, ${ }^{129}$ Xe NMR spectra in pure perfluoroalkanes allow a detailed analysis of the liquid structure, continuing that previously reported for the liquid alkanes. It should be emphasised that nanosegregation is here observed in fluids governed exclusively by dispersion interactions, in contrast to other examples in which hydrogen bonding and polarity play important roles. Given its simplicity, this case study is thus prone to have a general impact in understanding the early mechanisms of segregation, phase separation and self-assembly.
\end{abstract}

\section{Introduction}

Nano-segregated fluids, of which nano-emulsions are a prime example, are interesting and important systems both from a fundamental and an applied point of view. The existence of mesoscale inhomogeneity in fully miscible mixtures of simple fluids is an intriguing phenomenon with significant implications in the solvation behaviour of mixtures and has recently prompted studies on various different systems. ${ }^{1,2}$

At the molecular level, nano-segregation stems from a subtle balance between "favourable" and "unfavourable" intermolecular interactions that, although able to overcome the tendency of phase separation, nevertheless allow the formation of discrete domains. In other words, understanding nano-segregation is a step towards controlling miscibility, self-assembly and ultimately solubilisation. The importance to many technologically and biologically important processes, in which solubility is often a limiting factor, is thus apparent. Drug efficacy, for example, is frequently severely limited by poor solubility. Nano-emulsions

\footnotetext{
${ }^{a}$ Centro de Quimica Estrutural, Instituto Superior Técnico, Universidade de Lisboa, 1049-001 Lisboa, Portugal. E-mail: efilipe@tecnico.ulisboa.pt

${ }^{b}$ Centro de Química de Évora, Escola de Ciências e Tecnologia,

Universidade de Évora, Rua Romão Ramalho, 59, 7000-671 Évora, Portugal

$\dagger$ Electronic supplementary information (ESI) available. See DOI: 10.1039/c8cp06509h
}

are potentially novel drug delivery systems, ${ }^{3}$ allowing sustained and controlled release of drugs and improving their efficacy.

Most common nano-segregated liquids are formed by a distribution of polar and apolar domains. The former are often aqueous, while the latter are almost always formed by alkyl chains. Hydrogen bonding between water molecules and hydrophobic effects play a central role in these systems. However, there is a different type of apolar medium, formed by perfluorinated chains, which are simultaneously hydrophobic and "organophobic". Mixtures of alkanes with perfluoroalkanes are perhaps the simplest systems that demonstrate this low affinity between perfluorinated and hydrogenated compounds. Formed by molecules of comparable shape and volume, at similar thermodynamic conditions, and governed solely by dispersion forces, mixtures of alkanes + perfluoroalkanes could be expected to be the ultimate example of an ideal mixture. On the contrary, they typically display large regions of liquid-liquid immiscibility, low-boiling azeotropes and very large and positive excess volumes and enthalpies. This unexpected behaviour has long been known and interpreted as a sign of abnormally weak intermolecular dispersion forces between hydrogenated and fluorinated chains, whose underlying physical causes remain unexplained.

Therefore, systems where fluorinated and hydrogenated chains coexist tend to display segregation. Indeed, different 
levels of organization and segregation have been observed, such as diverse liquid crystal phases in pure semifluorinated compounds, ${ }^{4}$ the formation of micelles of semifluorinated alkanes (SFA) in alkane or perfluoroalkane solvents, ${ }^{5}$ or small nano-segregated domains in ionic liquids. ${ }^{6,7}$ It is thus plausible that simple (alkane + perfluoroalkane) mixtures, above the upper critical solution temperature (UCST), already display a certain degree of nano-segregation, forming domains of different local compositions. It should be emphasised however, that in this case segregation is the result of a subtle balance between relatively weak dispersion forces. The possibility of nanosegregation in (alkane + perfluoroalkane) systems was suggested by molecular dynamics simulation results in previous work by our group ${ }^{8,9}$ and one of the objectives of the present study is to examine this hypothesis using a combination of ${ }^{129} \mathrm{Xe}$ NMR and molecular dynamics simulations.

The ${ }^{129} \mathrm{Xe}$ isotope (26\% natural abundance) is active in NMR and its large polarizability gives it very high sensitivity to the immediate molecular environment; the range of chemical shifts for atomic xenon is larger than $200 \mathrm{ppm}$, depending only on the solvent. Moreover, its intermolecular potential is similar to that of $n$-alkanes ${ }^{10}$ and being a spherical particle, it should cause only a negligible structural perturbation when dissolved in a liquid and used as an NMR probe. In recent work on the liquid structure of alkanes, ${ }^{11}$ an approach combining ${ }^{129} \mathrm{Xe}$ NMR and molecular dynamics simulations allowed us to show, among other effects, that xenon is preferentially solvated by the methyl groups of $n$-alkane molecules, suggesting that these liquids tend to create cavities near the ends of the molecules, an effect that is more pronounced for the longer $n$-alkanes. We have also demonstrated the utility of this approach when applied to more complex systems such as ionic liquids; ${ }^{12}$ in this case, ${ }^{129} \mathrm{Xe}$ NMR was able to identify changes in the liquid structure along a homologous series of ILs and, in combination with MD simulations, to demonstrate differences in the mobility and accessibility of xenon to the polar and nonpolar regions of the liquid structure. In a recent paper, Welton and co-workers ${ }^{13}$ have used ${ }^{129} \mathrm{Xe}$ NMR in combination with other experimental techniques (SAXS and PALS) to elucidate the structure of ionic liquid mixtures. Finally, Pines and co-workers ${ }^{14}$ have recently reported a new class of ${ }^{129} \mathrm{Xe}$ NMR contrast agents based on PFC nanoemulsions, and demonstrated their potential use in applications such as chemical sensing or molecular imaging.

A significant number of computer simulations of (alkane + perfluoroalkane) mixtures can be found in the literature. ${ }^{15-22}$ Using both united-atom and atomistic force fields, all these studies have shown that substantial reductions of the cross interaction energy parameter from the geometric mean value need to be used to reproduce the experimental properties of the mixtures (phase equilibria, cross virial coefficients, gas solubility and excess enthalpy). It is also known, however, that the large positive excess volumes of these mixtures are in general not reproduced by the simulations ${ }^{17,23}$ and that the mentioned reduction of the cross-interaction energy parameter has a negligible effect on the volume of mixing. In our opinion, ignoring the large excess volumes displayed by these mixtures is a major drawback of previous models. Indeed, in recent work, ${ }^{24}$ we demonstrated that an adequate description of the volumetric behaviour is crucial to an overall correct modelling of these systems. This can be achieved by optimizing both the size and energy parameters of the cross interaction between fluorinated and hydrogenated chains to simultaneously reproduce the excess enthalpies and volumes. This approach was already followed by Zhang and Siepmann ${ }^{19}$ for the united-atom TraPPE force field, and is here applied to a more detailed atomistic model.

In this work, ${ }^{129}$ Xe NMR spectra were obtained, as a function of temperature, for solutions of xenon in pure perfluoroalkanes and in (hexane + perfluorohexane) mixtures. As previously described, these mixtures display extremely large deviations from ideality, including liquid-liquid immiscibility. As the upper critical solution temperature (UCST) is approached, reorganization at the molecular level can thus be expected. Starting from a macroscopically homogeneous liquid, well above the UCST, a gradual increase of mutual segregation should take place, leading to the formation of gradually larger and more stable inhomogeneities, domains or clusters and finally to coexisting liquid-liquid domains (phase-separation). Our ${ }^{129} \mathrm{Xe}$ NMR results distinctively show that xenon is able to sense these gradual changes, occurring within its coordination sphere, i.e., at the molecular level. The same systems were studied by molecular dynamics simulations using an atomistic force field. The results are interpreted together in order to elucidate the local liquid structure of the pure and mixed solvents, and their preferential molecular interactions with xenon. Nano-segregation in the (alkane + perfluoroalkane) mixtures above the liquid-liquid critical temperature is demonstrated and quantified.

\section{Experimental}

Xenon gas (99.995\%, natural isotopic abundance) was purchased from Air Liquide. Perfluoropentane (F5) and perfluorononane (F9) were obtained from Apollo Scientific, with 97\% (85\% $n$-isomer) and 99\% purities, respectively; perfluorohexane (F6, 99\%), perfluorooctane $(\mathrm{F} 8,98 \%)$ and $n$-hexane (H6, 99\%) were obtained from Aldrich. All substances were used as received.

For the determination of the ${ }^{129} \mathrm{Xe}$ chemical shift in the pure solvents, gaseous xenon was bubbled through around $0.5 \mathrm{ml}$ of the liquid in a standard $5 \mathrm{~mm}$ NMR tube, for at least 1 minute using a PTFE capillary; the NMR tube was then capped and sealed with Parafilm. For the reference, a standard $5 \mathrm{~mm}$ NMR tube was filled with xenon (which is denser than air), at atmospheric pressure, and capped in a similar way. The H6 + F6 mixtures were prepared by weight, using a Mettler AE240 analytical balance, in $5 \mathrm{~mm}$ NMR tubes equipped with J-Young glass + PTFE valves to prevent evaporation; first, xenon was bubbled through an appropriate amount of H6 inside the NMR tube (followed by weighing), and then F6 was added in order to obtain the desired composition. A sealed capillary glass tube filled with deuterated methanol was introduced into 
the sample tubes, in order to allow the use of a deuterium frequency lock.

The spectra were obtained using a Bruker Avance III $500 \mathrm{MHz}$ spectrometer equipped with a broadband $5 \mathrm{~mm}$ probe. Radio frequency pulses with a duration of $5 \mu \mathrm{s}$, with an interpulse delay of $10 \mathrm{~s}$, were used to record each free induction decay (FID), without spinning the sample. Between 20 and 200 scans were typically necessary, at each experimental point, to obtain spectra with good signal-to-noise ratios, and an exponential line broadening of $5 \mathrm{~Hz}$ was applied to the recorded FIDs. The spectrum of the reference sample was obtained in the beginning and at the end of each day of measurements to ensure that no drift occurred during the experiments.

The temperature of the NMR probe was measured with its integrated thermocouple and digitally controlled using an electrical resistor to heat a flow of either liquid nitrogen vapour or cooled air, which passed around the sample tube. This system was calibrated (sample $T$ vs. set $T$ ) using a specially constructed platinum resistance thermometer (Pt 100) mounted inside an NMR tube, which allowed not only the precise determination of temperature but also an estimate of the time necessary to achieve thermal equilibrium in the sample after a change in the set temperature, as well as of the temperature stability. Both the accuracy and stability of the control system are expected to be better than $0.5 \mathrm{~K}$. Molar magnetic susceptibilities of the studied perfluoroalkanes were determined in this work according to the method of Frei and Bernstein. ${ }^{25}$ The measured values, as well as a detailed description of the procedure, can be found as ESI. $\dagger$

\subsection{Molecular dynamics}

Alkanes and perfluoroalkanes (PFA) were modelled according to the OPLS-AA force field of Jorgensen et al., ${ }^{26,27}$ and xenon was represented as a Lennard-Jones sphere, with the parameters proposed by Bohn et al. ${ }^{28}$ Molecular dynamics simulations were performed using the DL_POLY Classic ${ }^{29}$ code, in the $N p T$ ensemble with periodic boundary conditions in all directions. Temperature and pressure were maintained using the NoséHoover thermostat and barostat with coupling constants of 0.5 and 2 ps, respectively. A cut-off distance of $14 \AA$ was used for both non-bonded Lennard-Jones and electrostatic potentials, applying standard analytic tail corrections for the dispersive energy and virial coefficients and the Ewald summation method to the electrostatic interactions beyond the cut-off. All bonds involving hydrogen atoms were constrained to their equilibrium distances using the SHAKE algorithm, and the equations of motion were integrated in 2 fs time-steps.

The structure of the solutions of xenon in PFA was analysed from 20 ns long simulations of one xenon atom in between 80 to 120 solvent molecules, from which radial distribution functions were obtained. These simulations were run at $1 \mathrm{~atm}$ and at the same reduced temperature $T_{\mathrm{r}}=0.58$, in order to compare the behaviour of the systems in similar thermodynamic states.

For the simulations of ( $\mathrm{H} 6+\mathrm{F} 6)$ mixtures, a total of 256 molecules were used, and at least $5 \mathrm{~ns}$ of simulation time was used to average the desired properties. After establishing the fluorine-hydrogen binary interaction parameters, a single xenon atom was inserted in each simulation box using the Packmol software $^{30}$ and the radial distribution functions were obtained from 20 ns runs, after 1 ns of equilibration time. The simulation parameters were the same as above.

\section{Results and discussion}

\subsection{Xenon in pure perfluoroalkanes}

The measured chemical shift, $\delta_{\text {exp }}$, of a given nucleus can be regarded as the sum of the chemical shift in the isolated molecule, $\delta^{0}$, the effect of the bulk magnetic susceptibility of the solvent, $\delta_{\mathrm{b}}$, and the chemical shift due to intermolecular interactions, $\delta_{\mathrm{m}}$ (eqn (1)). The latter term, also named the medium shift, is the quantity more relevant to the present study.

$$
\delta_{\exp }=\delta^{0}+\delta_{\mathrm{b}}+\delta_{\mathrm{m}}
$$

${ }^{129} \mathrm{Xe}$ chemical shifts have been measured as a function of temperature for solutions of xenon in 4 linear perfluoroalkanes (perfluoropentane (F5), perfluorohexane (F6), perfluorooctane (F8) and perfluorononane (F9)) from the melting point of the solvent to either its boiling point or the temperature limit of the used NMR probe. The experimental values were then corrected for the effect of the bulk susceptibility of the solvent and referenced to the chemical shift of gaseous xenon extrapolated to zero pressure, as discussed in more detail in previous work. ${ }^{11}$ The calculated medium shifts are reported in Table 1 and shown in Fig. 1a. The bulk susceptibility corrections were calculated using the molar magnetic susceptibilities determined in this work and the experimental densities previously reported. $^{31}$

As can be seen, the ${ }^{129}$ Xe medium shifts are always lower than $100 \mathrm{ppm}$, which is a clear sign of very weak interactions between xenon and perfluoroalkanes. To the best of our knowledge, these are among the lowest chemical shifts reported for xenon in a liquid solvent. In $n$-alkanes, for example, ${ }^{129} \mathrm{Xe}$ medium shifts range between 130 and $200 \mathrm{ppm}$. The ${ }^{129} \mathrm{Xe}$ medium shift measured in liquid ethane ${ }^{32}$ at $296 \mathrm{~K}$ (orthobaric conditions) has a value of $93 \mathrm{ppm}$, which falls within the range of values measured in the present work. However, ethane in those

Table $1{ }^{129}$ Xe medium shifts $\left(\delta_{\mathrm{m}}\right)$ in perfluoroalkanes

Perfluoropentane Perfluorohexane Perfluorooctane Perfluorononane

\begin{tabular}{lllllllll}
\hline$T / \mathrm{K}$ & $\delta_{\mathrm{m}} / \mathrm{ppm}$ & $T / \mathrm{K}$ & $\delta_{\mathrm{m}} / \mathrm{ppm}$ & $T / \mathrm{K}$ & $\delta_{\mathrm{m}} / \mathrm{ppm}$ & $T / \mathrm{K}$ & $\delta_{\mathrm{m}} / \mathrm{ppm}$ \\
\hline 175.4 & 98.8 & 238.7 & 85.3 & 254.9 & 86.0 & 287.2 & 79.9 \\
198.0 & 93.8 & 249.4 & 83.1 & 260.3 & 85.0 & 293.0 & 78.8 \\
220.7 & 88.2 & 260.3 & 80.1 & 281.8 & & 303.5 & 77.0 \\
238.7 & 82.7 & 271.6 & $78.8^{a}$ & 293.2 & 77.3 & 325.0 & 72.8 \\
243.3 & 82.4 & 281.8 & 75.4 & 303.5 & 75.7 & & \\
254.9 & 78.7 & 290.9 & 73.4 & 325.0 & 71.6 & & \\
266.0 & 77.0 & 292.3 & $74.1^{a}$ & & & & \\
288.7 & 71.5 & 303.5 & 70.8 & & & & \\
& & 313.0 & $69.3^{a}$ & & & & &
\end{tabular}

${ }^{a}$ Results used in the analysis of the (H6 + F6) mixture. 

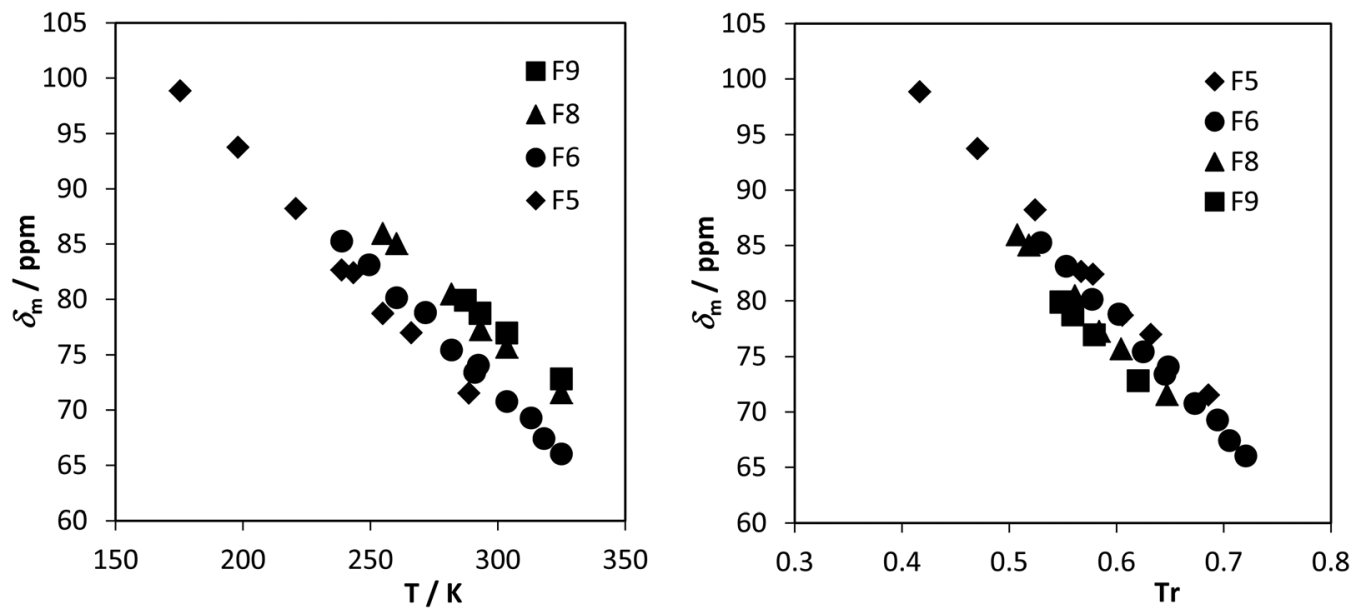

Fig. $1{ }^{129} \mathrm{Xe}$ medium shifts in perfluoroalkanes (left) as a function of temperature and (right) as a function of the reduced temperature of the solvent.

conditions is very close to its critical point $\left(T_{\mathrm{r}}=0.97\right)$ and has an extremely low liquid density, unlike the solvents now studied.

The Xe medium shifts in PFA decrease linearly with temperature for each substance, following the decrease of density and, at constant temperature, increase with the chain length of the solvent. In a previous paper we demonstrated how important it is to compare the behaviour of different systems at similar thermodynamic conditions. As an attempt to do so, the same ${ }^{129} \mathrm{Xe}$ medium shifts are also shown in Fig. 1 as a function of the reduced temperature $\left(T_{\mathrm{r}}=T / T_{\mathrm{c}}\right)$ of the solvent. As can be seen, the values for each solvent at the same $T_{\mathrm{r}}$ become closer, but seem to have a larger dispersion than was observed for the $n$-alkanes. ${ }^{11}$ This behaviour, already seen in other physical properties, may be related to differences in the structure of the solvent in the vicinity of xenon when the chain length increases. However, it should be considered that at least part of the effect might come from the presence of isomeric impurities in the used perfluoroalkanes; as was seen for alkanes, xenon dissolved in different isomers can have very different chemical shifts.

Xe chemical shifts depend on the number, nature, distance and orientation of the groups within xenon's coordination sphere. The average number and distance of groups can be calculated from the solvent molar density, ${ }^{31}$ assuming a uniform distribution of the groups throughout the liquid. The measured ${ }^{129}$ Xe medium shifts are shown in Fig. 2 as a function of the mean molar density of carbon atoms for each solvent. Using this representation, we can compare the interactivity between xenon and the $\mathrm{CF}_{2}$ and $\mathrm{CF}_{3}$ groups of perfluoroalkanes, assuming that the local density of the solvent in the vicinity of xenon atoms is equal to the mean bulk density and that the xenon atoms are randomly distributed within the solvent, i.e., no specific locations are preferred by the solute.

In this representation the ${ }^{129} \mathrm{Xe}$ shifts in different solvents form a series of straight parallel lines with positive slope. This supports the assumption that, for a given solvent, the density of shielding groups in the close vicinity of xenon is linearly related to the bulk density. It can now be seen that the shorter

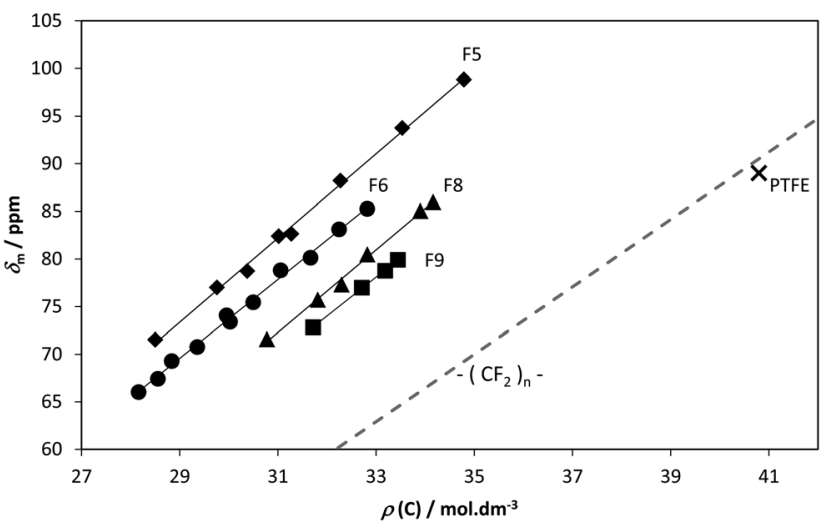

Fig. $2{ }^{129}$ Xe medium shifts in perfluoroalkanes, as a function of the mean molar density of carbon atoms of the solvent. The dashed line represents the estimated xenon medium shift in a perfluoroalkane formed exclusively of $\mathrm{CF}_{2}$ groups and $x$ is the experimental ${ }^{129} \mathrm{Xe}$ medium shift in PTFE. ${ }^{34}$

perfluoroalkanes, which have a larger proportion of $\mathrm{CF}_{3}$ groups, are more interactive towards xenon than the longer ones, in which the $\mathrm{CF}_{2}$ groups predominate. Moreover, there is a regular evolution of the xenon medium shift with the increase in the size of the perfluoroalkane, allowing the correlation of this quantity with the molar density of $\mathrm{CF}_{2}$ and of $\mathrm{CF}_{3}$ groups in the solution; a multilinear regression yields $\delta_{\mathrm{m}}=5.673 \rho\left(\mathrm{CF}_{3}\right)+$ $3.539 \rho\left(\mathrm{CF}_{2}\right)-53.876 \mathrm{ppm}$, which reproduces the observed medium shifts with a standard deviation of $0.5 \mathrm{ppm}$. This correlation allows us to calculate the medium shift of xenon in any $n$-perfluoroalkane, providing its density is known.

The same correlation was used to predict the chemical shift of xenon in poly(tetrafluoroethylene) (PTFE or Teflon), which can be regarded as an infinite perfluoroalkane formed only of $\mathrm{CF}_{2}$ groups. This prediction is shown in Fig. 2 as a dashed line. At this temperature PTFE is a solid, formed by variable proportions of crystalline and amorphous regions. It can be assumed that the experimentally measured ${ }^{129} \mathrm{Xe}$ chemical shift is only due to xenon atoms dissolved in amorphous regions whose structure is probably comparable to a liquid perfluoroalkane. 

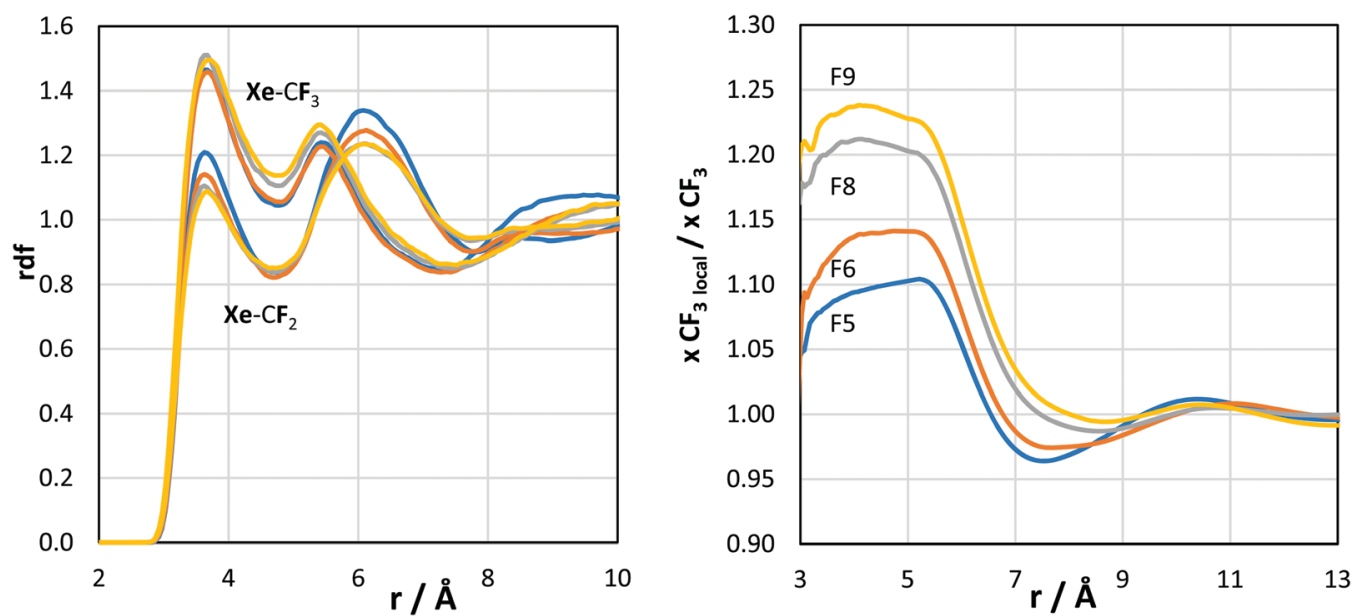

Fig. 3 (left) Xenon-fluorine radial distribution functions for the studied PFA solutions; (right) ratio between the local fraction of - $\mathrm{CF}_{3}$ fluorine atoms in the coordination sphere of xenon and their global molar fraction, as a function of the radius of the sphere.

Using an estimate of the density of pure amorphous PTFE, ${ }^{33}$ the experimental Xe chemical shift in PTFE at $298 \mathrm{~K}^{34}$ is also included in Fig. 2 and, as can be seen, the agreement with our prediction is very good.

Links between the observed chemical shifts and the structure of xenon's coordination shell (in terms of number, distance, and arrangement of groups) can be established through molecular dynamics simulations, by examining the radial distribution functions between xenon and the two types of fluorine atoms of the different solvents $\left(\mathrm{F}\right.$ in $\mathrm{CF}_{3}$, and $\mathrm{F}$ in $\mathrm{CF}_{2}$ ). The results are shown in Fig. 3(left).

As can be seen, the rdfs between xenon and the fluorine atoms of $\mathrm{CF}_{3}$ groups are more intense at shorter distances, up to around 5.5 $\AA$, meaning that the close vicinity of the xenon atom is enriched in $\mathrm{CF}_{3}$ groups, relative to the mean density. This suggests that, similarly to what was observed for Xe in $n$-alkanes, the small solute is preferentially solvated by the ends of the solvent chains. Between the studied perfluoroalkanes the differences are subtler, as could be expected due to their similar sizes. Nevertheless, it can be observed that the $\mathrm{Xe}-\mathrm{CF}_{3}$ peaks increase with the chain length of the PFA whereas the $\mathrm{Xe}-\mathrm{CF}_{2}$ peaks decrease, showing that the enrichment in $\mathrm{CF}_{3}$ groups becomes more important as the chain length of the solvent grows. This effect is clearly seen in Fig. 3(right), where the ratio between the local fraction of fluorine atoms belonging to a $\mathrm{CF}_{3}$ group and their bulk molar fraction is represented as a function of the radius of xenon's coordination sphere. In all the studied PFA solvents, and up to a coordination radius of more than $6.5 \AA$, Xe is surrounded by more $\mathrm{CF}_{3}$ groups than could be expected from their bulk ratio, and this enrichment grows with increasing chain length.

\subsection{Xenon in (hexane + perfluorohexane) mixtures}

${ }^{129}$ Xe NMR spectra were also obtained for mixtures of (hexane + perfluorohexane) (H6 + F6) as a function of temperature and composition. Since this mixture separates into two liquid phases at temperatures close to room temperature, great care was taken to ensure that the samples were introduced into the spectrometer above the upper critical solution temperature (UCST). Moreover, the spectra were obtained sequentially for each sample, in a path of descending temperature, and the phase separation was detected by an evident and abrupt change in the variation of the ${ }^{129} \mathrm{Xe}$ chemical shift with temperature. For the solution with the composition $x_{\mathrm{F} 6}=0.375$, two ${ }^{129} \mathrm{Xe}$ NMR peaks were observed below the de-mixing temperature, since the interface between both liquid phases was located inside the measurement region of the spectrometer probe used.

Chemical shifts of ${ }^{129}$ Xe dissolved in pure hexane were also measured in the present work, in the range of temperatures used for the (H6 + F6) mixtures. Although the obtained values compare very well with those in our previous work, ${ }^{11}$ we considered that the results for the pure components used in this part of the study should be from the same batch of solvents that was used to prepare the mixtures. This approach has been chosen since the xenon chemical shift can be very sensitive to small amounts of isomeric impurities present in the samples, which can slightly vary from one batch to the other. For the same reason, only the points obtained for the batch of perfluorohexane used in the preparation of the (H6 + F6) mixtures (identified in Table 1) were considered in this section. The full set of experimental results is reported in Table 2 and displayed in Fig. 4.

The first striking feature of Fig. 4 is the shape of the liquid-liquid coexistence phase diagram of ( $\mathrm{H} 6+\mathrm{F} 6)$, which is immediately apparent. Above the UCST, in the one-phase region, for each composition, the ${ }^{129} \mathrm{Xe}$ chemical shift varies with temperature in an essentially linear fashion, following the change in solvent density. By lowering the temperature, the mixture reaches the liquid-liquid phase envelope and the chemical shifts start to follow the change in composition of the coexisting phases. From this figure, the UCST was estimated to be $293 \mathrm{~K}$, in good agreement with the range of UCSTs ( $294 \mathrm{~K}$ to $296 \mathrm{~K}$ ) reported in the literature. ${ }^{35-41}$

In order to further analyse the results, the experimental chemical shifts were referenced to the resonance of gaseous 
Table 2 Uncorrected chemical shifts ( $\boldsymbol{\delta}_{\text {exp }}$ ) for ${ }^{129}$ Xe dissolved in hexane and in hexane + perfluorohexane mixtures

\begin{tabular}{|c|c|c|c|c|c|}
\hline \multicolumn{2}{|c|}{$n$-Hexane $\left(x_{\mathrm{F} 6}=0.0\right)$} & \multicolumn{2}{|c|}{$x_{\mathrm{F} 6}=0.199$} & \multicolumn{2}{|c|}{$x_{\mathrm{F} 6}=0.375$} \\
\hline$T / \mathrm{K}$ & $\delta_{\text {exp }} / \mathrm{ppm}$ & $T / \mathrm{K}$ & $\delta_{\text {exp }} / \mathrm{ppm}$ & $T / \mathrm{K}$ & $\delta_{\text {exp }} / \mathrm{ppm}$ \\
\hline 271.6 & 165.6 & $276.8^{a}$ & 151.3 & $271.6^{a}$ & $154.8 ; 90.4$ \\
\hline 292.3 & 158.9 & $284.0^{a}$ & 144.9 & $282.0^{a}$ & $146.7 ; 94.2$ \\
\hline \multirow[t]{14}{*}{313.0} & 152.2 & $289.2^{a}$ & 138.2 & $287.1^{a}$ & $141.0 ; 97.9$ \\
\hline & & $290.3^{a}$ & 136.3 & $289.2^{a}$ & $137.9 ; 100.3$ \\
\hline & & 291.3 & 134.4 & $291.3^{a}$ & $133.8 ; 104.0$ \\
\hline & & 292.3 & 134.1 & $292.8^{a}$ & $128.3 ; 109.4$ \\
\hline & & 293.4 & 133.6 & $293.4^{a}$ & 122.5 \\
\hline & & 294.4 & 133.3 & 293.9 & 118.0 \\
\hline & & 295.4 & 132.9 & 294.4 & 117.8 \\
\hline & & 297.5 & 132.2 & 295.4 & 117.4 \\
\hline & & 302.7 & 130.4 & 296.5 & 116.9 \\
\hline & & 307.9 & 128.6 & 297.5 & 116.5 \\
\hline & & 313.0 & 126.8 & 302.7 & 114.5 \\
\hline & & 318.2 & 125.0 & 307.9 & 112.7 \\
\hline & & & & 313.0 & 110.8 \\
\hline & & & & 318.2 & 109.0 \\
\hline
\end{tabular}

\begin{tabular}{|c|c|c|c|c|c|}
\hline \multicolumn{2}{|c|}{$x_{\mathrm{F} 6}=0.497$} & \multicolumn{2}{|c|}{$\underline{x_{\mathrm{F} 6}}=0.512$} & \multicolumn{2}{|c|}{$x_{\mathrm{F} 6}=0.745$} \\
\hline$T / \mathrm{K}$ & $\delta_{\exp } / \mathrm{ppm}$ & $T / \mathrm{K}$ & $\delta_{\exp } / \mathrm{ppm}$ & $T / \mathrm{K}$ & $\delta_{\exp } / \mathrm{ppm}$ \\
\hline $276.8^{a}$ & 91.8 & 294.4 & 106.5 & $273.7^{a}$ & 91.0 \\
\hline $284.0^{a}$ & 95.3 & 295.4 & 106.1 & $277.8^{a}$ & 92.3 \\
\hline $288.2^{a}$ & 98.7 & 296.0 & 105.9 & $278.9^{a}$ & 92.7 \\
\hline $291.3^{a}$ & 103.5 & 296.5 & 105.7 & 279.9 & 92.6 \\
\hline $292.3^{a}$ & 106.3 & 297.5 & 105.4 & 282.0 & 92.0 \\
\hline 293.4 & 108.4 & 298.5 & 104.9 & 287.1 & 90.4 \\
\hline 294.4 & 108.0 & 299.6 & 104.6 & 292.3 & 88.8 \\
\hline 297.5 & 106.8 & 300.6 & 104.2 & 297.5 & 87.2 \\
\hline 302.7 & 104.9 & 302.7 & 103.5 & 302.7 & 85.7 \\
\hline 307.9 & 103.1 & 303.7 & 103.1 & 307.9 & 84.2 \\
\hline 313.0 & 101.2 & 306.8 & 102.0 & 313.0 & 82.6 \\
\hline \multirow[t]{3}{*}{318.2} & 99.5 & 307.9 & 101.7 & 318.2 & 81.1 \\
\hline & & 313.0 & 99.9 & & \\
\hline & & 318.2 & 98.2 & & \\
\hline
\end{tabular}

${ }^{a}$ Points below the phase separation temperature. The values correspond to the ${ }^{129} \mathrm{Xe}$ chemical shift in one of the coexisting phases. xenon at zero pressure and corrected for the effect of the bulk susceptibility of the (H6 + F6) mixture. For this binary solvent, and assuming that the concentration of xenon is very low, the bulk susceptibility term can be calculated as:

$$
\delta_{\mathrm{b}}=-\left(\frac{4 \pi}{3}\right)\left([\mathrm{H} 6] \chi_{\mathrm{m}, \mathrm{H} 6}+[\mathrm{F} 6] \chi_{\mathrm{m}, \mathrm{F} 6}\right)
$$

where $\chi_{\mathrm{m}, \mathrm{H} 6}$ and $\chi_{\mathrm{m}, \mathrm{F} 6}$ are the molar magnetic susceptibilities and [H6] and [F6] are the molar concentrations of hexane and perfluorohexane. These were calculated from the pure component densities ${ }^{31,42}$ and the temperature dependent excess volumes of this system. ${ }^{35}$

Luhmer and Bartik proposed ${ }^{43}$ that the dependence of the xenon medium shift on the density of a solvent can be explicitly taken into account by defining the molar medium shift as $\delta_{\mathrm{m}}{ }^{*}=\delta_{\mathrm{m}} / \rho$ (with $\rho$ as the molar density in moles per litre, identical to the concentration in molarity units). The medium shift of xenon in a mixture can thus be calculated as the sum of the products of the molar medium shifts in the pure solvents and the respective molar densities in the mixture. In the case of our binary mixture, the xenon medium shift can be expressed as

$$
\delta_{\mathrm{m}, \text { predicted }}=[\mathrm{H} 6] \delta_{\mathrm{m}, \mathrm{H} 6}{ }^{*}+[\mathrm{F} 6] \delta_{\mathrm{m}, \mathrm{F} 6}{ }^{*}
$$

where $\delta_{\mathrm{m}, \mathrm{H} 6}{ }^{*}$ and $\delta_{\mathrm{m}, \mathrm{FG}}{ }^{*}$ are the molar medium shifts calculated for xenon in the pure solvents. It should be noticed that both $\delta_{\mathrm{m}}{ }^{*}$ have a small linear dependence on temperature, which was taken into account in the following analysis.

Because this equation assumes that the local proportion of both components in the coordination sphere of xenon is the same as their bulk concentration, the chemical shifts calculated in this way are the predicted chemical shifts for a random mixture, where there is no preferential solvation of xenon. Following the same line of reasoning, any differences between the calculated and the experimental medium shifts

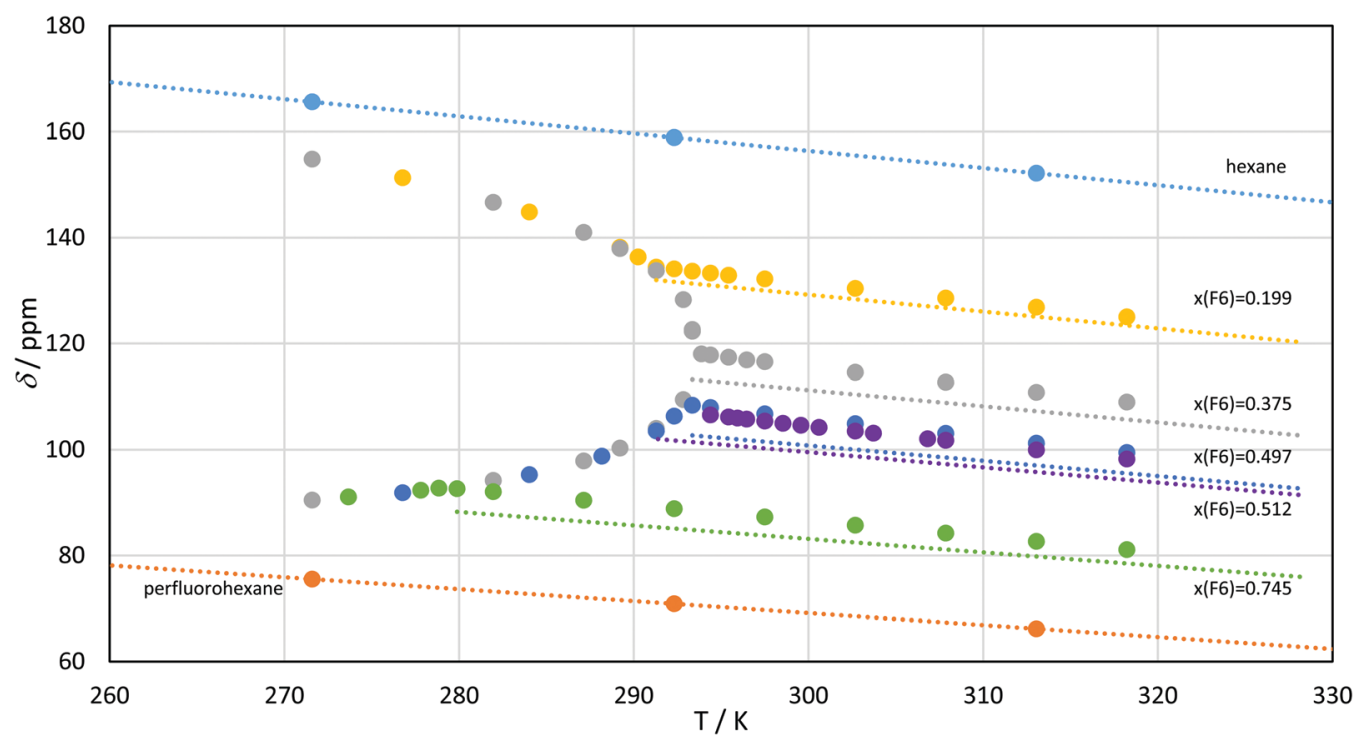

Fig. 4 Chemical shifts for ${ }^{129}$ Xe dissolved in $n$-hexane + perfluorohexane mixtures. Points represent the experimental values. The lines were predicted for each mole fraction from the molar medium shifts of the pure components (eqn (3)). 
indicate that the local concentration within xenon's coordination sphere is different from the nominal composition for the mixture and a sign of local enrichment in one of the components.

The ${ }^{129}$ Xe medium shifts predicted by eqn (3) are also presented in Fig. 4 as dashed lines. As can be seen, the experimental points for all the mixtures fall above the predicted lines, demonstrating that the average coordination sphere of xenon is always richer in hexane than the nominal composition of the mixture. The enrichment can be quantified using the same eqn (3) to calculate the composition that corresponds to each experimentally measured medium shift. These values can be identified as the average local compositions in the vicinity of the dissolved xenon atoms.

The differences between the calculated local compositions within xenon's coordination sphere and the nominal (or bulk) compositions of the solvent mixtures are shown in Fig. 5. As can be seen, these differences are negative for all the mixtures at all temperatures. Again this demonstrates that the xenon atoms are located in an environment which has a lower proportion of the fluorinated component than the bulk and, conversely, that xenon is preferentially solvated by the hydrogenated solvent. The effect of temperature is shown in Fig. 5a, where it is seen that the local compositions became closer to the nominal ones as the temperature increases, which could be expected since thermal agitation contributes to better mixing. In Fig. 5b it can be seen that the maximum enrichment is located at a composition close to equimolar. Nevertheless, the shape of the curves is skewed towards the higher compositions, suggesting that the effect is more pronounced when hexane is the minority in the mixture.

The structure of the (hexane + perfluorohexane) mixtures was further investigated by atomistic molecular dynamics simulations. It is well known that when modelling (alkane + perfluoroalkane) mixtures with either theoretical approaches or molecular simulations, a large reduction of the crossinteraction energy parameter $\left(\varepsilon_{12}\right)$ relative to the geometric mean combining rule (Berthelot's rule) is needed to reproduce the vapour-liquid and liquid-liquid equilibria, cross virial coefficients and excess enthalpy. ${ }^{18,19,21,44-50}$ However, as we have recently pointed out, ${ }^{8}$ even with a $10 \%$ reduction of the total unlike dispersive energy, the simulations fail to account for the large excess volumes displayed by these systems, which are of the order of $5 \mathrm{~cm}^{3} \mathrm{~mol}^{-1}$. This result indicates that the volumetric behaviour of (alkane + perfluoroalkane) mixtures is not only a consequence of weak dispersive interactions, but should also be connected to the repulsive component of the intermolecular potential. In the context of the OPLS-AA force field used in this work, which is based on the Lennard-Jones potential, this translates into the need for increasing the crosssize parameter $\left(\sigma_{12}\right)$ relative to the geometric mean rule value. Some alternative combining rules have been proposed, ${ }^{18,51}$ but none has been shown to provide a simultaneous successful description of the energetic and volumetric behaviour of mixtures of alkanes + perfluoroalkanes.

In this work, simulations have thus been performed optimizing both the energy $(\varepsilon)$ and size $(\sigma)$ parameters of the cross hydrogen-fluorine interaction to simultaneously match the experimental excess enthalpy and volume. The carbonhydrogen and carbon-fluorine cross parameters were kept at their original OPLS-AA values (geometric mean). We found that a $20 \%$ reduction of $\varepsilon_{\mathrm{H}-\mathrm{F}}$ and a $4 \%$ increase of $\sigma_{\mathrm{H}-\mathrm{F}}$ (relative to their corresponding geometric mean values) are needed to give simultaneous agreement between the simulated and experimental excess enthalpy and volume (Fig. S1, ESI $\dagger$ ). The simulations now quantitatively reproduce the excess volumes, including their temperature dependence, and good agreement with the experimental excess enthalpies is also achieved. In order to test transferability, simulations were also performed for the ( $n$-butane + perfluorobutane) equimolar mixture at $260 \mathrm{~K}$. The obtained excess volume $\left(4.5 \mathrm{~cm}^{3} \mathrm{~mol}^{-1}\right)$ and excess enthalpy (1.6 $\mathrm{kJ} \mathrm{mol}^{-1}$ ) also compare favourably with the experimental results of Simons and Mausteller ${ }^{52}\left(4.4 \mathrm{~cm}^{3} \mathrm{~mol}^{-1}\right.$ and $2.1 \mathrm{~kJ} \mathrm{~mol}^{-1}$, respectively).

Simulations were performed for systems consisting of one xenon atom dissolved in mixtures of (hexane + perfluorohexane) with different concentrations at $298 \mathrm{~K}$. Radial distribution functions were obtained from the simulated trajectories.

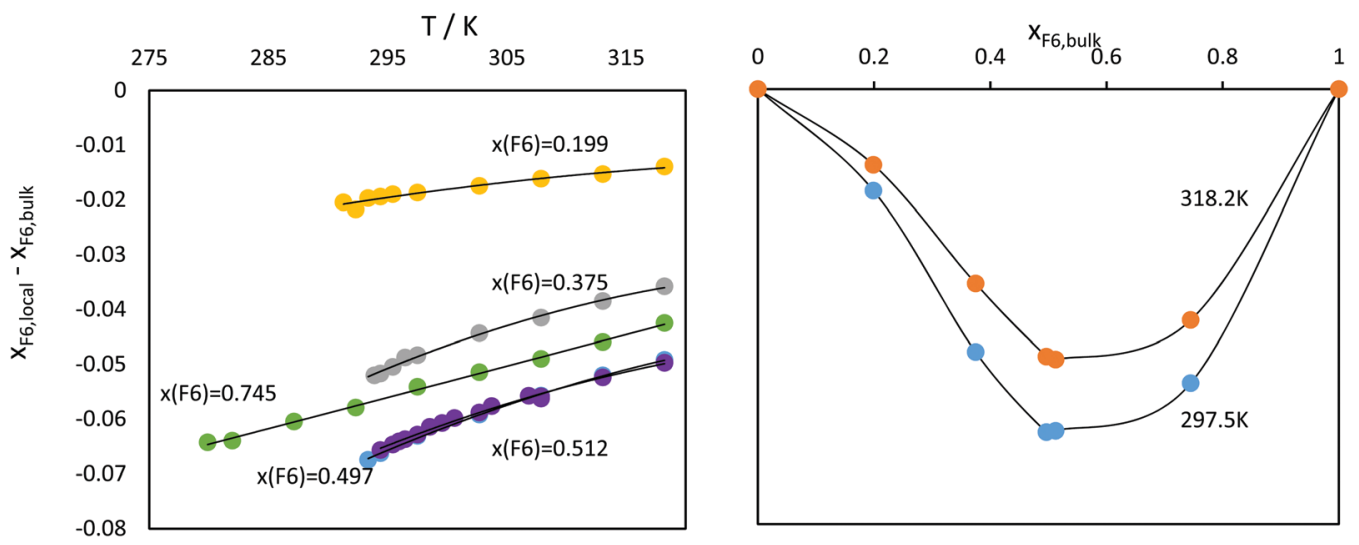

Fig. 5 Differences between the local composition within xenon's coordination sphere and the nominal composition of the studied mixtures: (a) as a function of temperature; (b) as a function of the bulk composition. 

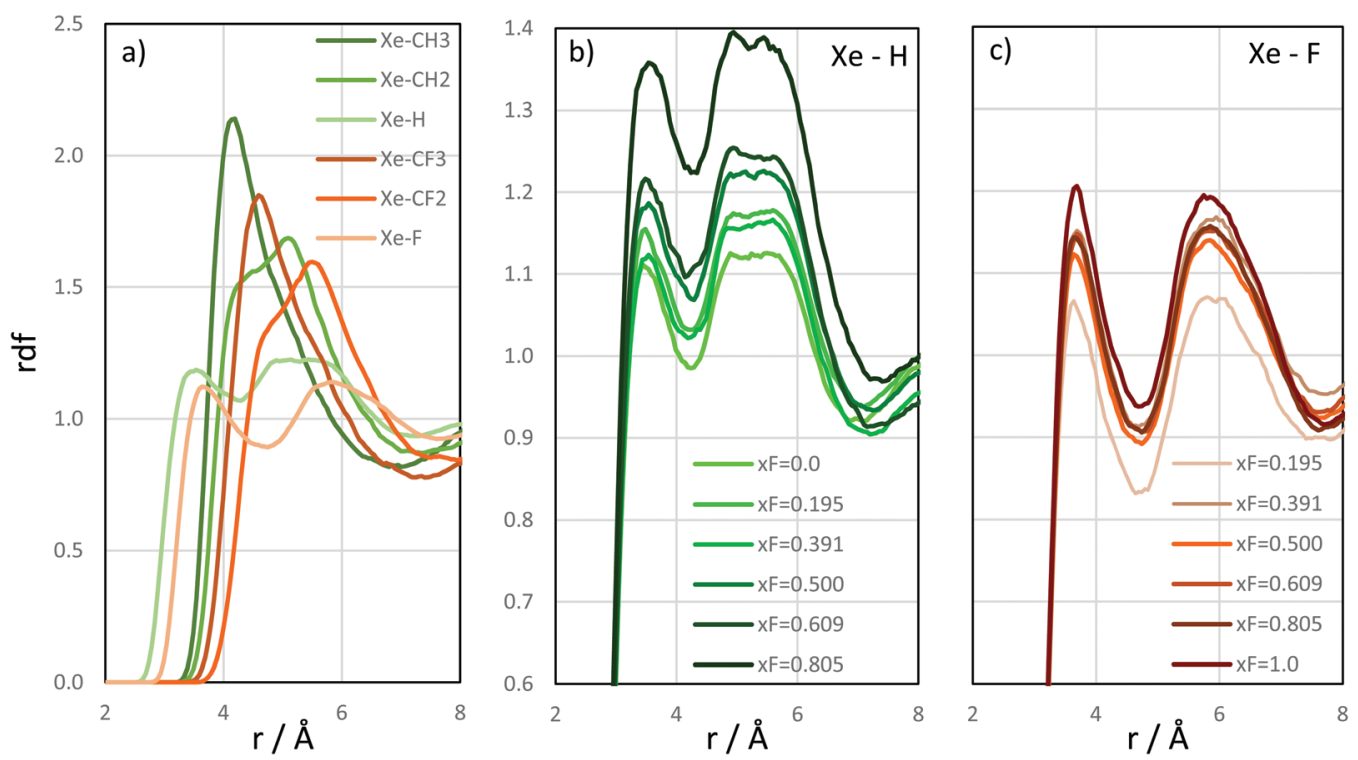

Fig. 6 (a) Radial distribution functions between xenon and the solvent atoms for the (hexane + perfluorohexane) equimolar mixture; (b) xenonhydrogen rdfs and (c) xenon-fluorine rdfs for (hexane + perfluorohexane) mixtures at different compositions.

For the equimolar mixture, the rdfs of the different solvent atoms around xenon are shown in Fig. 6a. It is evident that the rdfs involving hexane are significantly more intense than those involving perfluorohexane (hydrogen vs. fluorine, methyl carbon vs. perfluoromethyl carbon, and methylene carbon vs. perfluoromethylene carbon). This clearly demonstrates that xenon is sensitive to the nano-segregation between hydrogenated and fluorinated domains identified ${ }^{8,9}$ in previous work: when xenon is dissolved in an equimolar (hexane + perfluorohexane) mixture, it is preferentially found in environments richer in the hydrogenated component.

In Fig. $6 \mathrm{~b}$ and c, the xenon-hydrogen and xenon-fluorine rdfs are shown for the full range of compositions studied. As can be seen, the lowest xenon-hydrogen peak occurs in pure hexane and its intensity increases as hexane becomes more diluted. The opposite happens for xenon-fluorine, where the highest peak is seen in pure perfluorohexane and then decreases as the concentration of perfluorohexane becomes smaller. This is additional proof that xenon is preferentially located in the vicinity of hexane molecules: at high concentrations of hexane, xenon "avoids" the vicinity of perfluorohexane, originating the decrease of the Xe-F peak, whereas at low hexane concentrations xenon tends to stay close to the few hydrogenated molecules present, increasing the Xe-H peak. A more quantitative measure of the local composition of the coordination sphere of xenon was obtained by integrating the rdfs and then calculating the local fluorine mole fraction as a function of the radius of the coordination sphere. The values obtained for all the studied compositions, for distances between $4 \AA$ and $6 \AA$, are always lower than the bulk composition by between 0.02 and 0.06 in mole fraction units, in excellent agreement with those obtained from the analysis of the experimental results presented in Fig. $5 \mathrm{~b}$.

\section{Conclusions}

The liquid structure of four perfluoroalkanes (perfluoropentane, perfluorohexane, perfluorooctane and perfluorononane) was studied using ${ }^{129} \mathrm{Xe}$ as a NMR probe for the first time. Spectra were obtained as a function of temperature to allow the comparison of the different solvents at similar thermodynamic conditions. The results were rationalized in terms of the density, nature, and organization of the chemical groups within xenon's coordination sphere. It is clearly shown that the $\mathrm{CF}_{3}$ group is more interactive than $\mathrm{CF}_{2}$ and that xenon is not randomly distributed throughout the liquid, but preferentially located near the ends of solvent molecules, resulting in an enrichment of $\mathrm{CF}_{3}$ groups around xenon in comparison with the bulk proportion. Molecular dynamics simulations confirm all these hypotheses.

The performed analysis, together with a similar procedure recently reported for the $n$-alkanes, allows the prediction of the medium shift of xenon in alkanes, perfluoroalkanes and their mixtures at any composition and temperature, providing the density is known.

Liquid mixtures of (hexane + perfluorohexane) were also studied as a function of temperature. From the ${ }^{129} \mathrm{Xe}$ NMR spectra the liquid-liquid phase diagram is obtained in close agreement with literature results.

Experimental evidence was obtained indicating that mixtures of (hexane + perfluorohexane), above the upper critical solution temperature, segregate forming domains at the nanometric scale. The results suggest the presence of domains richer in the hydrogenated component, in which xenon "prefers" to be solvated. Conversely, this implies the existence of domains richer in the perfluorinated component.

The experimental results show that the average local concentration in the vicinity of xenon is 0.05 higher in hexane mole 
fraction than the nominal concentration of the mixture. Atomistic molecular dynamics simulations support this analysis in close agreement with the experimental data.

It should be emphasised that nano-segregation is here observed in fluids governed exclusively by dispersion interactions, in contrast to other examples in which hydrogen bonding and polarity play important roles. Given its simplicity, this example is thus prone to have a general impact in understanding the early mechanisms of segregation, phase separation and self-assembly.

\section{Conflicts of interest}

There are no conflicts to declare.

\section{Acknowledgements}

The authors thank Prof. Fernando Fernandes from Universidade de Lisboa for the generous lending of computing power, and acknowledge funding from Fundação para a Ciência e Tecnologia (FCT) through the project UID/QUI/0100/2013. PM acknowledges funding from FCT, postdoctoral grant SFRH/BPD/81748/2011.

\section{References}

1 M. Sedlák and D. Rak, Large-Scale Inhomogeneities in Solutions of Low Molar Mass Compounds and Mixtures of Liquids: Supramolecular Structures or Nanobubbles?, J. Phys. Chem. B, 2013, 117, 2495-2504.

2 T. N. Zemb, M. Klossek, T. Lopian, J. Marcus, S. Schöettl, D. Horinek, S. F. Prevost, D. Touraud, O. Diat and S. Marčelja, et al., How to Explain Microemulsions Formed by Solvent Mixtures without Conventional Surfactants, Proc. Natl. Acad. Sci. U. S. A., 2016, 113, 4260-4265.

3 Y. Singh, J. G. Meher, K. Raval, F. A. Khan, M. Chaurasia, N. K. Jain and M. K. Chourasia, Nanoemulsion: concepts, development and applications in drug delivery, J. Controlled Release, 2017, 252, 28-49.

4 C. G. Lux, B. Donnio, B. Heinrich and M. P. Krafft, Thermal Behavior and High- and Low-Temperature Phase Structures of Gemini Fluorocarbon/Hydrocarbon Diblocks, Langmuir, 2013, 29, 5325-5336.

5 P. Lo Nostro, Phase Separation Properties of Fluorocarbons, Hydrocarbons and Their Copolymers, Adv. Colloid Interface Sci., 1995, 56, 245-287.

6 Y. Shen, D. F. Kennedy, T. L. Greaves, A. Weerawardena, R. J. Mulder, N. Kirby, G. Song and C. J. Drummond, Protic Ionic Liquids with Fluorous Anions: Physicochemical Properties and Self-Assembly Nanostructure, Phys. Chem. Chem. Phys., 2012, 14, 7981-7992.

7 A. B. Pereiro, M. J. Pastoriza-Gallego, K. Shimizu, I. M. Marrucho, J. N. Canongia Lopes, M. M. Piñeiro and L. P. N. Rebelo, On the Formation of a Third, Nanostructured Domain in Ionic Liquids, J. Phys. Chem. B, 2013, 117, 10826-10833.
8 P. Morgado, A. R. Garcia, L. M. Ilharco, J. Marcos, M. Anastácio, L. F. G. Martins and E. J. M. Filipe, Liquid Mixtures Involving Hydrogenated and Fluorinated Alcohols: Thermodynamics, Spectroscopy, and Simulation, J. Phys. Chem. B, 2016, 120, 10091-10105.

9 P. Morgado, A. R. Garcia, L. F. G. Martins, L. Ilharco and E. J. M. Filipe, Alkane Coiling in Perfluoroalkane Solutions: A New Primitive Solvophobic Effect, Langmuir, 2017, 33, 11429-11435.

10 E. J. M. Filipe, L. M. B. Dias, J. C. G. Calado, C. McCabe and G. Jackson, Is Xenon an "ennobled" Alkane?, Phys. Chem. Chem. Phys., 2002, 4, 1618-1621.

11 P. Morgado, R. P. Bonifácio, L. F. G. Martins and E. J. M. Filipe, Probing the Structure of Liquids with ${ }^{129} \mathrm{Xe}$ NMR Spectroscopy: N-Alkanes, Cycloalkanes, and Branched Alkanes, J. Phys. Chem. B, 2013, 117, 9014-9024.

12 P. Morgado, K. Shimizu, J. M. S. S. Esperança, P. M. Reis, L. P. N. Rebelo, J. N. Canongia Lopes and E. J. M. Filipe, Using ${ }^{129}$ Xe NMR to Probe the Structure of Ionic Liquids, J. Phys. Chem. Lett., 2013, 4, 2758-2762.

13 N. J. Brooks, F. Castiglione, C. M. Doherty, A. Dolan, A. J. Hill, P. A. Hunt, R. P. Matthews, M. Mauri, A. Mele, R. Simonutti, I. J. Villar-Garcia, C. C. Weber and T. Welton, Linking the structures, free volumes, and properties of ionic liquid mixtures, Chem. Sci., 2017, 8, 6359-6374.

14 T. K. Stevens, R. M. Ramirez and A. Pines, Nanoemulsion Contrast Agents with Sub-picomolar Sensitivity for Xenon NMR, J. Am. Chem. Soc., 2013, 135, 9576-9579.

15 C. Hoheisel, Molecular-Dynamics Studies on Partially Miscible Binary-Liquid Mixture Methane-Tetrafluoromethane Based on Lennard-Jones (12-6) Potentials, Ber. Bunsenges. Phys. Chem., 1977, 81, 462-468.

16 M. Schoen and C. Hoheisel, Liquid $\mathrm{CH}_{4}$, Liquid $\mathrm{CF}_{4}$ and the Partially Miscible Liquid Mixture $\mathrm{CH}_{4} / \mathrm{CF}_{4}-\mathrm{A}$ Molecular Dynamics Study Based on Both a Spherically Symmetric and a Four-Centre Lennard-Jones Potential Model, Mol. Phys., 1986, 58, 699-709.

17 S. Brode and I. R. McDonald, Excess Thermodynamic Properties of Liquid Mixtures of Methane and Perfluoromethane, Mol. Phys., 1988, 65, 1007-1012.

18 W. Song, P. J. Rossky and M. Maroncelli, Modeling Alkane + perfluoroalkane Interactions Using All-Atom Potentials: Failure of the Usual Combining Rules, J. Chem. Phys., 2003, 119, 9145-9162.

19 L. Zhang and J. I. Siepmann, Pressure Dependence of the Vapor-Liquid-Liquid Phase Behavior in Ternary Mixtures Consisting of N-Alkanes, N-Perfluoroalkanes, and Carbon Dioxide, J. Phys. Chem. B, 2005, 109, 2911-2919.

20 J. J. Potoff and D. A. Bernard-Brunel, Mie Potentials for Phase Equilibria Calculations: Application to Alkanes and Perfluoroalkanes, J. Phys. Chem. B, 2009, 113, 14725-14731.

21 H. Dominguez, A. J. Haslam, G. Jackson and E. A. Müller, Modelling and Understanding of the Vapour-Liquid and Liquid-Liquid Interfacial Properties for the Binary Mixture of N-Heptane and Perfluoro-N-Hexane, J. Mol. Liq., 2013, 185, 36-43. 
22 H. Dominguez, The Non-Ideal Behaviour of the Interfacial Tension of the N-Heptane + Perfluoro- N-Hexane Mixture: A Computational Study, Chem. Phys. Lett., 2015, 627, 77-81.

23 P. Duarte, M. Silva, D. Rodrigues, P. Morgado, L. F. G. Martins and E. J. M. Filipe, Liquid Mixtures Involving Hydrogenated and Fluorinated Chains: (P, P, T, X) Surface of (Ethanol $+2,2,2$-Trifluoroethanol), Experimental and Simulation, J. Phys. Chem. B, 2013, 117, 9709-9717.

24 P. Morgado, J. Black, J. B. Lewis, C. R. Iacovella, C. McCabe, L. F. G. Martins and E. J. M. Filipe, Viscosity of Liquid Systems Involving Hydrogenated and Fluorinated Substances: Liquid Mixtures of (Hexane + perfluorohexane), Fluid Phase Equilib., 2013, 358, 161-165.

25 K. Frei and H. J. Bernstein, J. Chem. Phys., 1962, 37, 1891-1892.

26 W. L. Jorgensen, D. S. Maxwell and J. Tirado-Rives, Development and Testing of the OPLS All-Atom Force Field on Conformational Energetics and Properties of Organic Liquids, J. Am. Chem. Soc., 1996, 118, 11225-11236.

27 E. K. Watkins and W. L. Jorgensen, Perfluoroalkanes: Conformational Analysis and Liquid-State Properties from Ab Initio and Monte Carlo Calculations, J. Phys. Chem. A, 2001, 105, 4118-4125.

28 M. Bohn, S. Lago, J. Fischer and F. Kohler, Excess Properties of Liquid Mixtures from Perturbation Theory: Results for Model Systems and Predictions for Real Systems, Fluid Phase Equilib., 1985, 23, 137-151.

29 W. Smith, T. R. Forester and I. T. Todorov, The DL_POLY Classic User Manual, Daresbury Laboratory, UK, 2012.

30 L. Martínez, R. Andrade, E. G. Birgin and J. M. Martínez, PACKMOL: A Package for Building Initial Configurations for Molecular Dynamics Simulations, J. Comput. Chem., 2009, 30, 2157-2164.

31 P. Morgado, J. B. Lewis, C. M. C. Laginhas, L. F. G. Martins, C. McCabe, F. J. Blas and E. J. M. Filipe, Systems Involving Hydrogenated and Fluorinated Chains: Volumetric Properties of Perfluoroalkanes and Perfluoroalkylalkane Surfactants, J. Phys. Chem. B, 2011, 115, 15013-15023.

32 Y. H. Lim, N. E. Nugara and A. D. King, NMR Chemical Shifts of Xenon-129 in Liquid Carbon Dioxide, Nitrous Oxide, Ethane, and Propane at $23^{\circ} \mathrm{C}$, J. Phys. Chem., 1993, 97, 8816-8819.

33 P. J. Rae and D. M. Dattelbaum, The properties of poly(tetrafluoroethylene) (PTFE) in compression, Polymer, 2004, 45, 7615-7625.

34 Y. Wang, P. T. Inglefield and A. A. Jones, NMR characterization of penetrants in high permeability polymers, Polymer, 2002, 43, 1867-1872.

35 R. G. Bedford and R. D. Dunlap, Solubilities and Volume Changes Attending Mixing for the System: Perfluoro- $n$ hexane- $n$-Hexane, J. Am. Chem. Soc., 1958, 80, 282-285.

36 W. J. Gaw and R. L. Scott, Volume Changes in the Critical Solution Region, J. Chem. Thermodyn., 1971, 3, 335-345.
37 T. E. Block, N. F. Judd, I. A. McLure, C. M. Knobler and R. L. Scott, Excess Volumes in the Critical Solution Region, J. Phys. Chem., 1981, 85, 3282-3290.

38 P. J. Clements, S. Zafar, A. Galindo, G. Jackson and I. A. McLure, Thermodynamics of Ternary Mixtures Exhibiting Tunnel Phase Behaviourk. 3. Hexane-HexamethyldisiloxanePerfluorohexane, J. Chem. Soc., Faraday Trans., 1997, 93, 1331-1339.

39 C. Duce, M. R. Tiné, L. Lepori and E. Matteoli, VLE and LLE of Perfluoroalkane + Alkane Mixtures, Fluid Phase Equilib., 2002, 199, 197-212.

40 R. A. Khairulin, S. V. Stankus and V. A. Gruzdev, LiquidLiquid Coexistence Curve of $n$-Perfluorohexane- $n$-Hexane System, Int. J. Thermophys., 2007, 28, 1245-1254.

41 H. Matsuda, A. Kitabatake, M. Kosuge, K. Kurihara, K. Tochigi and K. Ochi, Liquid-liquid Equilibrium Data for Binary Perfluoroalkane (C6 and C8) + n-Alkane Systems, Fluid Phase Equilib., 2010, 297, 187-191.

42 I. Cibulka, Saturated Liquid Densities of 1-Alkanols from C1 to C10 and N-Alkanes from C5 to C16: A Critical Evaluation of Experimental Data, Fluid Phase Equilib., 1993, 89, 1-18.

43 M. Luhmer and K. Bartik, Group Contribution Analysis of Xenon NMR Solvent Shifts, J. Phys. Chem. A, 1997, 101, 5278-5283.

44 R. L. Scott, The Anomalous Behavior of Fluorocarbon Solutions, J. Phys. Chem., 1958, 62(2), 136-145.

45 C. McCabe, A. Galindo, A. Gil-Villegas and G. Jackson, Predicting the High-Pressure Phase Equilibria of Binary Mixtures of PerfluoroE- $n$-Alkanes $+n$-Alkanes Using the SAFT-VR Approach, J. Phys. Chem. B, 1998, 102(41), 8060-8069.

46 C. Colina, A. Galindo, F. J. Blas and K. E. Gubbins, Phase Behavior of Carbon Dioxide Mixtures with -Alkanes and -Perfluoroalkanes, Fluid Phase Equilib., 2004, 222-223, 77-85.

47 P. Morgado, C. McCabe and E. J. M. Filipe, Modelling the Phase Behaviour and Excess Properties of Alkane + Perfluoroalkane Binary Mixtures with the SAFT-VR Approach, Fluid Phase Equilib., 2005, 228-229, 389-393.

48 J. D. Haley and C. McCabe, Predicting the Phase Behavior of Fluorinated Organic Molecules Using the GC-SAFT-VR Equation of State, Fluid Phase Equilib., 2017, 440, 111-121.

49 S. Aparicio, Phase equilibria in perfluoroalkane plus alkane binary systems from PC-SAFT equation of state, J. Supercrit. Fluids, 2008, 46, 10-20.

50 F. R. Varanda, L. F. Vega, J. A. P. Coutinho and I. M. Marrucho, Liquid-liquid equilibrium of substituted perfluoro- $n$-octane $+n$-octane systems, Fluid Phase Equilib., 2008, 1-2, 85-89.

51 A. J. Haslam, A. Galindo and G. Jackson, Prediction of Binary Intermolecular Potential Parameters for Use in Modelling Fluid Mixtures, Fluid Phase Equilib., 2008, 266(1-2), 105-128.

52 J. H. Simons and J. W. Mausteller, The Properties of N-Butforane and Its Mixtures with N-Butane, J. Chem. Phys., 1952, 20, 1516-1519. 